\title{
Agroindustrial Waste As Potential Source for Obtaining Nanocellulose
}

\section{Residuos Agroindustriales Como Fuente Potencial Para La Obtención de Nanocelulosa}

\author{
Jorge Silva-Yumi ${ }^{1,2}$, Wendy Peralta Holguin ${ }^{1,2}$, and Carlos Medina Serrano ${ }^{1,2}$ \\ ${ }^{1}$ Escuela Superior Politécnica de Chimborazo, Facultad de Ciencias, Química, Riobamba, Ecuador \\ ${ }^{2}$ Grupo de Investigación en Materiales Avanzados, Riobamba, Ecuador
}

I International Congress of Science and Technology Morona Santiago-CICTMS 2020

Corresponding Author:

Jorge Silva-Yumi

jorge.silvay@espoch.edu.ec

Published: 29 August 2021

Production and Hosting by

Knowledge E

(c) Jorge Silva-Yumi et al. This article is distributed under the terms of the Creative Commons Attribution License, which permits unrestricted use and redistribution provided that the original author and source are credited.

\section{Abstract}

This review focuses on evaluating the potential of agricultural residues produced in Ecuador (rice, banana, corn, sugar cane, pineapple) as raw material for obtaining nanocellulose. A review of published documents regarding the obtaining and applications of nanocellulose was carried out, as well as the characteristics of agro-industrial waste and production in Ecuador. Nanocellulose constitutes a versatile nanomaterial, it can be applied in areas such as health, food, engineering, environmental, electronics, etc. The physical-chemical characteristics of each of the residues derived from the production of rice, banana, corn, sugar cane, pineapple allow us to conclude in the first instance that Ecuador has a potential in the generation of matter for obtaining nanocellulose, it is However, it is necessary to develop basic and applied research that allows a use and exploitation of these wastes with a greater added value than that currently obtained.

Keywords: Cellulose, nanocellulose, nanocrystals, agricultural residues.

\section{Resumen}

La presente revisión se enfoca en evaluar la potencialidad de residuos agrícolas producidos en Ecuador (arroz, plátano, maíz, caña de azúcar, piña) como materia prima para la obtención de nanocelulosa. Se realizó una revisión de documentos publicados respecto a la obtención y aplicaciones de nanocelulosa, así como, de las características de los residuos agroindustriales y la producción en Ecuador. La nanocelulosa constituye un nanomaterial versátil, se puede aplicar en áreas como la salud, alimentos, ingeniería, ambiental, electrónica, etc. Las características físico químicas de cada uno de los residuos derivados a partir de la producción de arroz, plátano, maíz, caña de azúcar, piña permite concluir en primera instancia que Ecuador tiene un potencial en la generación de materia para la obtención de nanocelulosa, es necesario sin embargo el desarrollo de investigación básica y aplicada que permita un uso y aprovechamiento de estos residuos con un mayor valor agregado que el que se obtiene actualmente.

Palabras Clave: Celulosa, nanocelulosa, nanocristales, residuos agrícolas. 


\section{Introducción}

En Ecuador el sector agrícola se considera uno de los principales de la economía nacional, pese a esta contribución, la generación de residuos por parte de la agroindustria genera consecuencias ambientales [1].

Debido a las características químicas y biológicas que poseen los residuos agroindustriales, estos se pueden utilizar para diversas aplicaciones como obtención de productos de consumo o extracción de compuestos. Sin embargo, Ecuador no registra un aprovechamiento que genere valor a este tipo de residuos [1]

La extracción de celulosa; un polímero de base biológica [1], desde residuos agroindustriales, a partir de la cual se puede obtener nanocelulosa (NC); un material que tiene un amplio número de aplicaciones: películas, reforzamiento de polímeros, fibras, textiles, en biomedicina, en electrónica, entre otras [2], puede constituir un aporte al crecimiento económico del país.

La obtención de nanocelulosa a escala industrial a partir de residuos agrícolas, para producir productos de valor agregado es aún un campo de investigación y exploración [3] a nivel mundial y aún más en Ecuador.

El objetivo de esta investigación es analizar si los residuos agroindustriales producidos en Ecuador pueden constituir una fuente potencial para la obtención de nanocelulosa.

\section{Metodología}

Los artículos empleados fueron seleccionados respecto a las características de los residuos agroindustriales, volúmenes de producción, procesos de obtención y aplicaciones de nanocelulosa.

Se inicia con la definición de nanoceluosa y los métodos utilizados en su obtención, posteriormente se revisa algunas de las aplicaciones de la NC en áreas como medicina, electrónica, ambiente, alimentos. Finalmente se analiza el potencial de los residuos agroindustriales producidos en Ecuador como materia prima para la obtención de nanocelulosa.

\subsection{Nanocelulosa}

La NC es un material celulósico con al menos una de sus dimensiones comprendida dentro de la escala nanométrica (1-100 nm). Existen tres tipos de nanocelulosa: celulosa bacteriana, celulosa nanocristalina y celulosa nanofibrilada [4].

Los diferentes tipos de NC exhiben distintas propiedades que dictan su aplicabilidad y funcionalidad, es decir, ciertos tipos de nanocelulosa son más adecuadas que otros en aplicaciones específicas [5].

Los nanocristales de celulosa son partículas rígidas en forma de varilla que consisten de segmentos de celulosa en una estructura cristalina casi perfecta [6]; poseen diámetros de 3-20 nm [2] y longitudes de 100-1000 nm [7] aunque varían en sus 
dimensiones según la fuente [2]. En comparación con la celulosa macro, que tiene mayores fracciones amorfas, estos nanocristales, exhiben alta resistencia específica, alta área superficial y propiedades cristalinas líquidas únicas [6].

La NC posee una área superficial reactiva con grupos hidroxilo específicos [2] que permiten modificaciones [5] como: sustitución [8], oxidación, esterificación, eterificación, injerto de polímeros [5], etc., que facilitan interacciones con otros compuestos que le otorgan propiedades adicionales.

La NC se puede obtener a través de dos enfoques: de arriba hacia abajo o de abajo hacia arriba por biosíntesis.

El enfoque de arriba hacia abajo consiste en la desintegración de la fibra mediante tratamientos químicos o mecánicos o una combinación de ambos [9], el tratamiento de blanqueo por ejemplo consiste en eliminar la mayor parte de lignina y otros componentes hasta obtener holocelulosa que incluye principalmente hemicelulosa y celulosa [10]. La hidrólisis ácida por otra parte involucra la digestión de celulosa, dilución y purificación [11].

El enfoque de abajo hacia arriba a través de biosíntesis incluye la fermentación de azúcares de bajo peso molecular mediante el uso de bacterias [9].

\subsection{Aplicaciones}

La NC ha surgido como un material sostenible con propiedades como: biocompatibilidad, baja densidad, alto módulo específico, excelente estabilidad en disolventes, baja toxicidad y abundancia natural $[12,13]$ que la hacen apta para aplicaciones que van desde el área de la salud, alimentos, ingeniería, ambiental, hasta la electrónica.

Las solicitudes de patentes sobre NC incluyen materiales compuestos, absorbentes, papel y cartón, productos alimenticios, revestimientos de papel y cartón, cosméticos, artículos de tocador y materiales filtrantes [11].

En el área electrónica la investigación se ha orientado hacia su utilización como polímero en componentes electrónicos por su flexibilidad y baja expansión térmica [14]. Al no ser conductora se ha trabajado en enfoques de modificación química o física para preparar materiales conductores [15].

En el área de alimentos, la NC sirve para el desarrollo de materiales compuestos que pueden inhibir el crecimiento de bacterias y hongos con el fin de desarrollar embalajes activos y biodegradables [16].

En el área médica la NC sirve para el desarrollo de hidrogeles biodegradables y no tóxicos que promueven la regeneración celular y que por tanto podrían ser utilizados en vendaje de heridas y reparación de cartílagos [17].

La NC se puede utilizar para la obtención de películas que constituyen una nueva clase de papel con propiedades mejoradas que integra el componente de sostenibilidad [18]. Mediante modificaciones estas películas a base de NC podrían ser utilizadas en el desarrollo de pantallas táctiles, celdas solares y nanogeneradores [19]. 
En el área ambiental la NC puede ser utilizada para el desarrollo de membranas para el tratamiento de agua debido a su biodegradabilidad y bajo coste, la NC puede constituir la membrana o puede incorporarse en otras como aditivo [20].

La inclusión de una cantidad mínima de NC en una matriz de polímero podría mejorar las características mecánicas del material, en comparación con la matriz del polímero simple [13].

En respuesta a los desafíos energéticos y responsabilidad ambiental, la NC constituye un material versátil para el desarrollo de dispositivos de almacenamiento de energía electroquímica, la NC puede actuar como matriz o como nanorelleno [21].

Las mejoras en su producción y propiedades como: alta área superficial, comportamiento reológico, absorción de agua y ausencia de propiedades citotóxicas facilitan su uso como agente estabilizador, ingrediente alimentario funcional y en envases de alimentos [22].

\subsection{Rediduos}

\subsubsection{Agroindustriales}

Los residuos o subproductos agroindustriales se generan en cualquier proceso de producción y no poseen utilidad posterior en la cadena de producción [23], son las partes derivadas de los cultivos que no son destinados al consumo humano, entre los que se incluyen: tallos, hojas, cáscaras, semillas, etc., que provienen de cereales, frutas, legumbres entre otros productos [24].

Los residuos agroindustriales son de naturaleza predominantemente sólida y orgánica, rica en polímeros de celulosa y hemicelulosa (entre 75\% y 80\%) [23].

Pequeñas cantidades de los residuos agroindustriales son aprovechados para alimento de animales, obtención de energía por procesos de combustión, preparación de abonos orgánicos, entre otras, sin embargo, la mayor parte de estos son quemados en los campos abiertos causando contaminación ambiental en medios abióticos, bióticos [24] además de problemas socioeconómicos [23].

Se revisan a continuación las características de los principales productos cultivados en Ecuador y de los cuales se derivan varios residuos producto de su cosecha y utilización.

\section{(i) Arroz}

Es un cereal que se obtiene a partir de la planta Oryza sativa [3] que pertenece a la familia de las gramíneas, considerado el alimento básico para más de la mitad de la población mundial [25].

En el proceso de cosecha se obtiene 1 tonelada de paja de arroz por cada 8 toneladas cultivadas de este cereal [26], luego en el proceso de pilado se obtiene: arroz pilado, polvillo, cascarilla, arrocillo en un $65.3 \%, 9 \%, 23.2 \%$ y $2.5 \%$ respectivamente [27]. 
La cascarilla de arroz tiene una naturaleza fuerte, leñosa y abrasiva; algunas de las características se muestran en la Tabla 1.

\section{Table 1}

Carcaterísticas de la cáscara y paja de arroz según diferentes autores.

\section{Característica}

Densidad $\left(\mathrm{g} / \mathrm{cm}^{3}\right)$

Densidad aparente $\left(\mathrm{g} / \mathrm{cm}^{3}\right)$

Humedad (\%)

$\mathrm{pH}$ (suspensión)

$\mathrm{SiO}_{2}(\%)$

Materia Orgánica total (\%)

Relación C/N (\%)

$\mathrm{N}(\%)$

Lignina (\%)

Cenizas (\%)

$\mathrm{P}_{2} \mathrm{O}_{3}$ (\%)

$\mathrm{P}_{2} \mathrm{O}_{5}$ (\%)

$\mathrm{K}_{2} \mathrm{O}(\%)$

$\mathrm{Pb}(\mathrm{mg} / \mathrm{kg})$

$\mathrm{Cd}(\mathrm{mg} / \mathrm{kg})$

$\mathrm{Cr}(\mathrm{mg} / \mathrm{kg})$

Fuente:

\begin{tabular}{llll} 
& & Cantidad & \\
- & - & 1.56 \\
- & - & 0.017 \\
12.7 & - & 8.6 \\
- & - & 7.00 \\
14.3 & - & - \\
80.3 & - & 84.9 \\
- & - & 39.5 \\
0.93 & - & 1.03 \\
- & $3.2-4.4$ & - \\
\hline- & $16-18$ & - \\
\hline 0.1 & - & - \\
\hline 1.55 & - & 0.32 \\
0.75 & - & 2.46 \\
\hline 0.12 & - & - \\
6.0 & - & - \\
{$[26]$} & {$[28]$} & - \\
\hline
\end{tabular}

\section{(ii) Plátano}

El plátano pertenece a la familia de las Musáceas, nativa de Asia, dentro de esta familia están: Musa Cavendish (bananos) y Musa paradisíaca (plátanos).

En la cosecha de los bananos y plátanos solo se utiliza del 20 al 30\% de su biomasa dejando un 70 a $80 \%$ de residuos sin aplicaciones: el pseudo tallo y raquis o tallo floral [30], hojas, entre otras. La Tabla 2 muestra algunas de las características del pseudo tallo.

\section{(iii) Maíz}

El maíz, Zea mays L. es una planta herbácea anual, nativa del hemisferio norte, originaria de México y se cultiva en todo el mundo [34]. Las mazorcas, las hojas y los tallos son residuos importantes del procesamiento y consumo de maíz. En la Tabla 3 se puede apreciar características de la hoja de maíz. 
Table 2

Características del pseudo tallo de plátano.

\begin{tabular}{|c|c|c|c|c|c|c|}
\hline \multirow{2}{*}{$\begin{array}{l}\text { Característica } \\
\text { Humedad (\%) }\end{array}$} & \multicolumn{6}{|c|}{ Cantidad } \\
\hline & 10.00 & 9.74 & - & 9.74 & - & - \\
\hline Cenizas (\%) & 1.02 & 8.65 & 1.5 & 8.64 & 6.75 & 8.65 \\
\hline $\mathrm{Na}(\%)$ & - & - & - & - & 4.44 & - \\
\hline K (\%) & - & - & - & - & 9.44 & - \\
\hline $\mathrm{Ca}(\%)$ & - & - & - & - & 13.3 & - \\
\hline Mg (\%) & - & - & - & - & 2.55 & - \\
\hline $\mathrm{P}(\%)$ & - & - & - & - & 1.37 & - \\
\hline $\mathrm{Fe}(\%)$ & - & - & - & - & 0.03 & - \\
\hline Zn (\%) & - & - & - & - & 0.08 & - \\
\hline Mn (\%) & - & - & - & - & 0.01 & - \\
\hline Lignina (\%) & 5.10 & 15.07 & 18.6 & 15.07 & - & 19.4 \\
\hline Celulosa (\%) & 63.20 & 31.21 & 63.90 & 31.26 & - & 37.8 \\
\hline Hemicelulosa (\%) & 18.60 & 14.98 & 1.3 & 14.98 & - & 26.6 \\
\hline Fuente: & [31] & [31] & [31] & [31] & {$[32]$} & [33] \\
\hline
\end{tabular}

Table 3

Características de la hoja de mazorca de maíz.

Característica

$\mathrm{pH}$

$\mathrm{N}$ (ppm)

Cenizas (\%)

$\mathrm{K}(\mathrm{meq} / 100 \mathrm{ml})$

$\mathrm{S}$ (ppm)

$P(p p m)$

Ca (meq/100ml)

$\mathrm{Mg}(\mathrm{meq} / 100 \mathrm{ml})$

Lignina (\%)

Holocelulosa (\%)

Celulosa (\%)

Fuente:

\begin{tabular}{lcc} 
& Cantidad \\
- & 5.86 \\
- & 20 \\
0.761 & - & 0.21 \\
- & 17 \\
- & 83 \\
- & 2.5 \\
- & 0.61 \\
- & - \\
\hline 23.00 & - \\
78.86 & - \\
43.14 & {$[35]$} \\
{$[34]$} &
\end{tabular}

\section{(iv) Caña de azúcar}

La caña de azúcar, Saccharum officinarum, es una gramínea originaria de la India [36] a partir de esta se obtiene azúcar además de otros tipos de endulzantes y como subproducto el bagazo. Las características del bagazo se pueden visualizar en la Tabla 4. 
Table 4

Características del bagazo de la caña de azúcar según varios autores.

\begin{tabular}{|c|c|c|c|c|c|}
\hline \multirow{2}{*}{$\begin{array}{l}\text { Característica } \\
\text { Humedad (\%) }\end{array}$} & \multicolumn{5}{|c|}{ Cantidad } \\
\hline & - & - & 4 & - & - \\
\hline Carbono Fijo (\%) & - & 41.9 & $28.7-30.7$ & - & - \\
\hline Cenizas (\%) & $1.5-5.0$ & 11.74 & 1.26 & 0.51 & - \\
\hline C (\%) & - & 42.54 & 48.58 & 23.48 & - \\
\hline $\mathrm{H}(\%)$ & - & 5.71 & 5.97 & 3.28 & - \\
\hline $\mathrm{N}(\%)$ & - & 0.63 & 0.20 & - & - \\
\hline $\mathrm{S}(\%)$ & - & 0.30 & - & - & - \\
\hline $\mathrm{O}(\%)$ & - & 39.62 & 38.98 & 23.23 & - \\
\hline Lignina (\%) & $19-24$ & - & - & - & $18-26.3$ \\
\hline Holocelulosa (\%) & $59-76$ & - & - & - & - \\
\hline Celulosa (\%) & 32.44 & - & - & - & $41-55$ \\
\hline Hemicelulosa (\%) & - & - & - & - & $20-27.5$ \\
\hline Fuente: & [37] & [38] & [39] & [40] & [41] \\
\hline
\end{tabular}

\section{(v) Piña}

La piña (Ananas comosus) es una fruta tropical y exótica, durante el procesamiento de esta fruta los desechos de las hojas y cáscaras corresponden a aproximadamente el $50 \%$ del peso total [42]. La Tabla 5 y 6 muestran las características de la hoja y cáscara de piña respectivamente.

\section{Table 5}

Carcaterísticas de la hoja de piña.

Característica
Ceniza (\%)
Pectina (\%)
Lignina (\%)
Celulosa (\%)
Hemicelulosa (\%)
Fuente:

Cantidad
7.37
2.32
13.88
43.53
21.88
$[43]$

En Ecuador los cultivos de arroz, maíz, plátano y caña de azúcar predominan la superficie de hectáreas cultivadas al 2020, los porcentajes en relación al total de áreas cultivadas se pueden visualizar en la Figura 1.

La nanocelulosa puede obtenerse a partir de una amplia variedad de fuentes de celulosa, como madera, fibras, pastos, animales marinos, algas, hongos, invertebrados y bacterias [11]. 
Table 6

Carcaterística de la cáscara de piña.

Característica
Ceniza (\%)
Pectina (\%)
Lignina (\%)
Celulosa (\%)
Hemicelulosa (\%)
Fuente:

\begin{tabular}{lc} 
& Cantidad \\
1.5 & \\
2.49 & 10 \\
10.01 & 74 \\
40.55 & \\
28.69 & {$[44]$} \\
\hline 43$]$ &
\end{tabular}

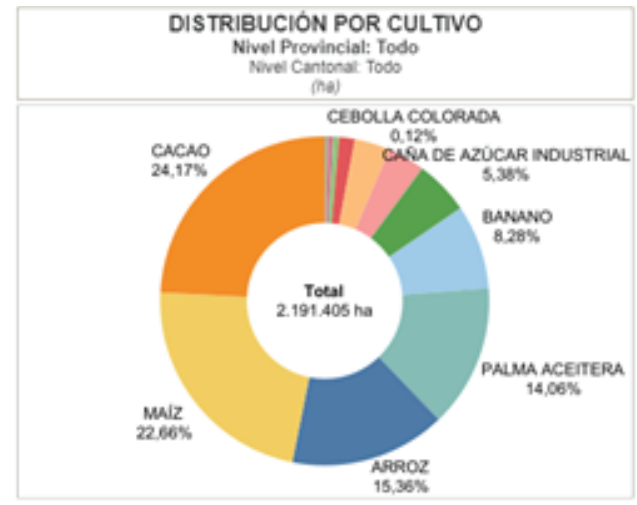

Figure 1

Distribución de producción de cultivos en Ecuador. (Fuente: [45].)

El arroz es el cultivo más extenso e importante del Ecuador pues aprovecha más de la tercera parte de la superficie cultivable [25], con una producción aproximada de 1,350,093 Tm. [46].

Se ha obtenido nanocristales de celulosa mediante hidrólisis ácida a partir de paja de arroz con rendimiento de $90.28 \%$ [47], se ha utilizado también cáscara de arroz, observándose diferencias en tamaño, cristalinidad, resistencia y estabilidad térmica, en función de la variedad de arroz utilizada [48].

La producción mundial de bananas es aproximadamente de 105 millones de toneladas al año, en Ecuador la producción estimada corresponde a 6,505,635 Tm [49].

Ecuador produce aproximadamente 1,618, 519 Tm [50] de maíz que genera una cantidad de biomasa residual que fluctúa entre las 20 y 35 toneladas por hectárea y en el maíz de choclo varía de 16 a 25 toneladas por hectárea [34]. Onkarappa et al. Obtuvieron nanocelulosa a partir de maíz mediante hidrólisis ácida [51].

La producción mundial de caña de azúcar alcanza aproximadamente 1.450 millones de toneladas con 22 millones de hectáreas de cultivo, es un cultivo muy importante, que va en ascenso debido al incremento poblacional [52]. En Ecuador la producción corresponde a $7.502 \mathrm{Tm}$ [53].

La piña en Ecuador registra una producción para exportación de 80.000 Tm [54]. 
Se ha obtenido también NC a partir de cascarilla de arroz, paja de arroz, fibra y pseudo tallo del plátano, tronco de palma aceitera, bagazo de caña de azúcar [3, 7, 9], piña [42] y hojas de maíz [55], lo que respalda que los residuos de estos productos pueden ser aprovechados para la obtención de NC.

De las características de los residuos agrícolas revisadas en las Tablas 1, 2, 3, 4, 5 y 6 y del volumen de producción de estos productos en Ecuador se puede decir que estos constituyen una fuente potencial para la obtención de NC.

\section{Conclusiones}

Las características de los residuos agroindustriales que muestran diversidad de contenido y concentraciones de materiales lignocelulósicos procedentes de la producción de arroz, azúcar, maíz, plátano y piña permiten concluir que son aptos para ser aprovechados en la obtención de nanocelulosa.

Ecuador cuenta con una potencial materia prima a ser valorizada que permitan un mayor rédito económico a los productores de estos recursos o la generación de empresas dedicadas al tratamiento de estos residuos para la obtención de nanocelulosa.

Es necesario impulsar el desarrollo de investigación básica y aplicada en torno a la obtención de nanocelulosa a partir de los residuos agroindustriales revisados en este artículo.

\section{Agradecimientos}

Al Grupo de Investigación en Materiales Avanzados de la ESPOCH.

\section{Complicaciones de Interéses}

No existen intereses particulares por parte de los autores o de la entidad científica que pudiesen afectar directa o indirectamente a los resultados.

\section{References}

[1] Riera MA, Maldonado S, Palma R. Residuos Agroindustriales generados en Ecuador para la Elaboracion de biplásticos. Rev Ing Ind. 2018;227-46.

[2] Pech SC. Obtención y modificación de nanocristales de celulosa de residuos de Agave Tequilana Weber para la producción de nanorefuerzos para Poli (ácido láctico). Centro de Investigación Científica de Yucatán; 2018.

[3] Kaur M, Kumari S, Sharma P. Chemically Modified Nanocellulose from rice husk: Synthesis and characterisation. AIR. 2018;13(3):1-11.

[4] Thomas P, Duolikun T, Rumjit NP et al. Comprehensive review on nanocellulose: Recent developments, challenges and future prospects. J Mech Behav Biomed Mater. 2020;110.

[5] Abitbol T, Rivkin A, Cao Y et al. Nanocellulose, a tiny fiber with huge applications. Curr Opin Biotechnol. 2016;39:76-88.

[6] Belitz H-D, Hans D, Grosch W, Schieberle P. Food chemistry. Springer-Verlag; 2009.

[7] Pereira B, Arantes V. Advances in sugarcane biorefinery: Technologies, commercialization, policy issues and paradigm shift for bioethanol and by-products. São Paulo: Elsevier; 2018. Nanocelluloses from sugarcane biomass; p. 179-96. 
[8] Castro F, Delgado F. La nanocelulosa: propiedades y aplicaciones. 2016.

[9] Ilyas RA, Sapuan SM, Ibrahim R et al. Production, processes and modification of nanocrystalline cellulose from agro-waste: A review. Nanocrystalline Mater. 2020;

[10] Phanthong P, Reubroycharoen P, Hao X, Xu G, Abudula A, Guan G. Nanocellulose: Extraction and application. Carbon Resour Convers. 2018;1(1):32-43.

[11] Blanco A, Monte MC, Campano C, Balea A, Merayo N, Negro C. Handbook of nanomaterials for industrial applications. Elsevier; 2018. Chapter, Nanocellulose for industrial use: Cellulose Nanofibers (CNF), Cellulose Nanocrystals (CNC), and Bacterial Cellulose (BC); p. 74-126.

[12] Jose J, Thomas V, Vinod V, Abraham R, Abraham S. Nanocellulose based functional materials for supercapacitor applications. J Sci Adv Mater Devices. 2019;4(3):333-40.

[13] Mishra RK, Sabu A, Tiwari SK. Materials chemistry and the futurist eco-friendly applications of nanocellulose: Status and prospect. J Saudi Chem Soc. 2018;22(8):949-78.

[14] Tayeb P, Tayeb AH. Nanocellulose applications in sustainable electrochemical and piezoelectric systems: A review. Carbohydr Polym. 2019;224(July):115149.

[15] Du X, Zhang Z, Liu W, Deng Y. Nanocellulose-based conductive materials and their emerging applications in energy devices - A review. Nano Energy. 2017;35:299-320.

[16] Maliha M, Herdman M, Brammananth R et al. Bismuth phosphinate incorporated nanocellulose sheets with antimicrobial and barrier properties for packaging applications. J Clean Prod. 2020;246:119016. https://doi.org/10.1016/j.jclepro.2019.119016

[17] Curvello R, Raghuwanshi VS, Garnier G. Engineering nanocellulose hydrogels for biomedica applications. Adv Colloid Interface Sci [Internet]. 2019;267:47-61. https://doi.org/10.1016/j.cis.2019.03. 002

[18] Mu R, Hong X, Ni Y et al. Recent trends and applications of cellulose nanocrystals in food industry. Trends Food Sci Technol. 2019;93(September):136-44.

[19] Fang Z, Hou G, Chen C, Hu L. Nanocellulose-based films and their emerging applications. Curr Opin Solid State Mater Sci. 2019;23(4):100764.

[20] Tan HF, Ooi BS, Leo CP. Future perspectives of nanocellulose-based membrane for water treatment. J Water Process Eng. 2020;37(May):101502.

[21] Guo R, Zhang L, Lu Y, Zhang X, Yang D. Research progress of nanocellulose for electrochemical energy storage: A review. J Energy Chem. 2020;51:342-61.

[22] Gómez H. C, Serpa A, Velásquez-Cock J et al. Vegetable nanocellulose in food science: A review. Food Hydrocoll. 2016;57:178-86.

[23] Vargas Y, Peréz L. Aprovechamiento de residuos agroindustriales en el mejoramiento de la calidad del ambiente. Rev Fac ciencias Básicas. 2018;1(1).

[24] Arias R, Meneses J. Caracterización residuos agroindustriales (cascarilla de arroz y cascarilla de café), como materia prima potencial para la obtención de bioetanol. Managua: Universidad Nacional Autónoma de Nicaragua; 2016.

[25] CFN. Ficha sectorial: Arroz. 2018.

[26] Abril D, Navarro E, Abril A. La paja de arroz. Consecuencias de su manejo y alternativas de aprovechamiento. Rev la Fac Agron. 2009;17:69-79.

[27] Vargas J, Alvarado P, Vega-Baudrit J, Porras M. Caracterización del subproducto cascarillas de arroz en búsqueda de posibles aplicaciones como materia prima en procesos. Rev Científica. 2013;23(1).

[28] Bartaburu D, Montes E, Pereira M. Utilización de la paja de arroz en la alimentación animal. Sitio Argentino Prod Anim

[29] UPU. Paja de arroz.

[30] Mazzeo M, Libardo L, Luis M, Guerrero L, Botero J. Aprovechamiento industrial de residuos de cosecha y poscosecha del plátano en el departamento de caldas. Rev Educ en Ing. 2010;128-39.

[31] Subagyo A, Chafidz A. IntechOpen. 2020. Banana nutrition - Function and processing kinetics. Banana pseudo-stem fiber: Preparation, characteristics, and applications.

[32] Thorat R, Bobade H. Utilization of banana pseudo stem in food applications. Int J Agric Eng. 2018;11:869.

[33] Carchi D. Aprovechamiento de los Residuos Agrícolas provenientes del cultivo de Banano para obtener nanocelulosa. Universidad de Cuenca; 2014.

[34] Prado-Martínez M, Anzaldo-Hernández J, Becerra-Aguilar B, Palacios-Juárez H, Vargas-Radilloo J, Rentería-Urquiza M. Caracterización de hojas de mazorca de maíz y de bagazo de caña para la elaboración de una pulpa celulósica mixta. Madera y Bosques. 2012;18(3):37-51.

[35] Guacho E. Caracterización Agro-morfológica del maíz (Zea mays L.). San José de Chazo: Escuela Superior Politécnica de Chimborazo; 2014. 
[36] Rosales C. Evaluación de tres frecuencias de riego con polietileno, en suelo arcilloso, sobre el crecimiento y rendimiento de caña de azúcar. Universidad Rafael Landívar; 2013.

[37] Prado K, Spinacé M. Isolation and characterization of cellulose nanocrystals from pineapple crown waste and their potential uses. Int J Biol Macromol. 2018;

[38] Antolin G, Oliva D. Caracterización del bagazo de caña de azúcar mediante Análisis Térmico. Inf Tecnológica. 2003;91-6.

[39] Manals-Cutiño E, Penedo-Medina M, Salas-Tort D. Caracterización del bagazo de caña como biomasa vegetal. Tecnología Química. 2015;

[40] Diaz R. Caracterización energética del bagazo de caña de azúcar del ingenio Valdez. Riobamba; 2008.

[41] Teixeira S, Arenales A, Eunice DA et al. Sugarcane bagasse: Applications for energy production and ceramic materials. J Solid Waste Technol Manag. 2015;41(3):229-38.

[42] Madureira A, Atatoprak T, Cabuk D, Sose F, Pullar R, Pintado M. Extraction and characterisation of cellulose nanocrystals from pineapple peel. Int Journalof Food Stud. 2018;7(April):24-33.

[43] Sánchez M, Ramos M, Mora R, Jimenéz E. Chemical characterisation of the industrial residues of the pineapple (ananas comosus). J Agric Chem Environ. 2014;3:53-56.

[44] TICSO. Aprovechamiento residuos biomasa de produccion de piña (ananas comosus) para municipio de aguazul casanare. 2014.

[45] Ganadería MA. Superficie de principales cultivos a nivel nacional.

[46] CFN. Ficha sectorial: Arroz. 2020.

[47] Thakur M, Sharma A, Ahlawat V, Bhattacharya M, Goswami S. Process optimization for the production of cellulose nanocrystals from rice straw derived $\alpha$-cellulose. Mater Sci Energy Technol. 2020;3:328-34.

[48] Rashid S, Dutta H. Characterization of nanocellulose extracted from short, medium and long grain rice husks. Ind Crops Prod. 2020;154(May):112627.

[49] CFN. Ficha sectorial: Bananos y plátanos. 2020.

[50] El comercio. Se fijó en \$14,60 el saco del maíz amarillo duro, \$0,65 menos que en 2019. 2020.

[51] Onkarappa HS, Prakash GK, Pujar GH, Rajith KCR, Radha V, Betageri VS. Facile synthesis and characterization of nanocellulose from Zea mays husk. Polym Compos. 2020;41(8):3153-9.

[52] Matute M. Caracterización físico-química del jugo de cinco variedades de caña de azucar (Saccharum officinarum) en la hacienda el Jardín. Escuela Superior Politécnica Agropecuaria de Manabí Manuel Félix López; 2016.

[53] CFN. Ficha sectorial: Azúcar. 2020.

[54] Espinoza MV. Ecuador exporta piña dulce a Europa y América. Líderes. 2017;8-10.

[55] Yang X, Han F, Xu C et al. Effects of preparation methods on the morphology and properties of nanocellulose (NC) extracted from corn husk. Ind Crops Prod. 2017;109(November):241-7. 